\title{
Carnets
}

Revue électronique d'études françaises de l'APEF

Deuxième série - $20 \mid 2020$

Imaginaire(s) du Voyage

\section{Voyage et nostalgie dans Onitsha et L'Africain de J.M.G Le Clézio}

\section{Mouhamadou Ngapout Kpoumié}

\section{(2) OpenEdition}

1 Journals

\section{Édition électronique}

URL : http://journals.openedition.org/carnets/12237

DOI : $10.4000 /$ carnets. 12237

ISSN : 1646-7698

Éditeur

APEF

\section{Référence électronique}

Mouhamadou Ngapout Kpoumié, « Voyage et nostalgie dans Onitsha et L'Africain de J.M.G Le Clézio », Carnets [En ligne], Deuxième série - 20 | 2020, mis en ligne le 30 novembre 2020, consulté le 20 avril 2021. URL : http://journals.openedition.org/carnets/12237 ; DOI : https://doi.org/10.4000/carnets. 12237

Ce document a été généré automatiquement le 20 avril 2021.

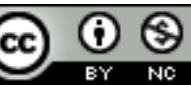

Carnets est mis à disposition selon les termes de la licence Creative Commons - Atribution - Pas d'utilisation commerciale 4.0 International. 


\title{
Voyage et nostalgie dans Onitsha et L'Africain de J.M.G Le Clézio
}

\author{
Mouhamadou Ngapout Kpoumié
}

\section{Introduction}

1 Chez Le Clézio, tout est mouvement, d'un territoire à un autre, avec une forte inscription des espaces réels. À leur contact, les individus développent des dispositions affectives, en sorte que l'expérience de l'espace se transforme en expérience de soi. Selon qu'elle est méliorative ou péjorative, ils peuvent nourrir de la nostalgie ou de la mélancolie. Sentiment de regret des temps passés ou des lieux disparus ou devenus lointains, auxquels on associe des sensations agréables, la nostalgie est provoquée par la perte ou le rappel des éléments passés, tant sur le plan temporel que spatial. L'Africain et Onitsha ${ }^{1}$ sont des récits mettant en scène des expériences migratoires en Afrique et en Europe à l'époque coloniale. Les voyageurs y sont en quête du territoire idéal sur lequel ils pourraient s'installer et s'épanouir. Quand bien même ils parviennent à se procurer du plaisir dans un ailleurs idyllique, ils doivent aussitôt le quitter pour revenir à la terre natale. Commence alors la détresse causée par l'éloignement et la rupture. La présente étude entend explorer les modalités, les jeux et les enjeux du voyage dans la construction des personnages. Pour ce faire, elle empruntera les considérations théoriques et pratiques de la géocritique, laquelle met l'accent sur l'analyse des espaces réels, pour établir leur "contribution à la détermination/indétermination des identités " (Westphal, 2000: 17). Après une présentation de l'itinéraire des voyageurs, l'analyse s'intéressera aux objets et traces constitutifs du paradis perdu, pour enfin montrer leur influence sur l'identité des voyageurs. 


\section{Itinéraires du voyage}

2 Dans Onitsha et L'Africain, la trajectoire des personnages est guidée par la quête de l'espace originel. Perçu, au départ, comme lieu exotique, où on va avec espérance de retour, le voyage vers ce lieu s'apparente à un retour chez soi. Dresser le carnet de voyage invite à découvrir les lieux traversés ou habités par les voyageurs. Dans les œuvres du corpus, ceux-ci quittent la France pour la côte ouest africaine, plus précisément à Ogoja et Onitsha. Conçu comme un déplacement d'un lieu à un autre, le voyage suppose un ensemble de motivations qui poussent le migrant à partir. La guerre et la promiscuité justifient ce départ qui s'apparente à une fuite. En fait, ayant choisi pour toile de fond des romans la période coloniale, et notamment la deuxième guerre mondiale, Le Clézio montre la situation précaire des habitants en France. Dans les deux récits, les protagonistes fuient ce pays où les séquelles de la guerre sont encore brûlantes, un pays où manquent l'argent et la joie de vivre. Ils embarquent pour le Cameroun anglais et le Nigéria, afin de commencer une nouvelle vie, une vie dont ils avaient rêvé, loin de Nice, loin de la guerre. Le narrateur d'Onitsha exprime le désir de recommencement manifesté par la famille Allen en ces mots : «Ils s'en allaient, jamais plus rien ne serait comme autrefois » $(0: 14)$. L'ailleurs devient un lieu rêvé, fantasmé par les personnages. Ils espèrent y trouver du bonheur, chose qui manque à leur vie en France.

3 Bien plus, le confinement - conséquence de la guerre qui sévit dans le pays - est l'une des raisons de ce déplacement. À cause de l'insécurité qui règne, les protagonistes ne peuvent sortir du domicile familial. Cet espace étroit, qui étouffe, crée chez eux une certaine claustrophobie, en sorte qu'il devient un cadre infernal. En témoignent ces propos de L'Africain :

Le présent africain effaçait tout ce qui l'avait précédé. La guerre, le confinement dans l'appartement de Nice (où nous vivions à cinq dans deux pièces mansardées, et même à six en comptant la bonne Maria dont ma grand-mère n'avait pas résolu de se passer), les rations ou bien la fuite dans la montagne où ma mère devait se cacher, de peur d'être raflée par la Gestapo (L'A : 16-17).

Manifestement, ce sont des personnages topophobes qui entendent quitter Nice pour un ailleurs où ils espèrent oublier leurs traumatismes existentiels. Curieusement, ceuxci trouveront en Afrique, notamment à Banso et Onitsha, respectivement dans L'Africain et Onitsha, le lieu de libération de soi. Le départ pour ces espaces devient donc le lieu de réalisation de leurs fantasmes. Ils embarquent pour le Nigéria afin de recommencer une nouvelle vie, une vie dont ils avaient rêvé. Dans Onitsha, les protagonistes quitteront Nice pour Onitsha. Avant ce voyage, ils n'ont eu quelques connaissances de cette localité que grâce aux lettres de Geoffroy Allen, le géniteur de Fintan, alors résidant dans cette ville portuaire du Nigéria. Les multiples escales du navire dans les différentes villes côtières permettent aux voyageurs de faire quelques promenades et d'entrer en contact avec la nature, le climat, de nouvelles personnes, bref un ensemble de découvertes qui servent de prélude à leur vie au Nigéria. Une fois arrivés à Onitsha, ces personnages s'adaptent aux modes de vie et au climat. Le séjour y est agréable pour la famille dans la mesure où chaque membre s'invente une passion: Geoffroy pour l'histoire du peuple de Méroë parti de l'ancienne Egypte, Fintan pour la liberté et la communion avec la nature, Maria Luisa pour le bonheur matrimonial retrouvé. Mais ces trois itinéraires seront perturbés par la révocation de Geoffroy Allen de la compagnie United Africa pour laquelle il travaille. Geoffroy manifeste son 
mécontentement avec véhémence à l'écoute de cette décision: "Je ne partirai pas. Nous avons le droit de rester ici quelques temps. (...) Et qui décide ça ? J'irai ailleurs, vers le nord, à Jos, à Kano » $(0: 179)$. Malgré ce refus, ces personnages doivent s'en aller à cause des déboires qui s'accumulent: la maladie de Geoffroy, la diminution des ressources alimentaires et financières, l'expulsion de la maison de service. Toutefois, même s'ils perdent les privilèges que leur donne la United Africa Company, du moins, ils ne se risqueront pas de perdre ce havre de paix et de joie.

Dans L'Africain, il s'agit du séjour du père du narrateur anonyme arrivé à Banso, à l'ouest du Cameroun, en 1928 et exerçant comme médecin, en provenance de Georgetown en Guyane (L'A : 49). Son long séjour dans la vaste région qu'il parcourt pour son travail l'amène à s'enraciner dans la culture indigène. Sa femme et ses enfants le rejoignent vingt ans après. Le narrateur, âgé de huit ans alors, y passe un séjour agréable dont les souvenirs constituent l'essentiel de ce récit : errance, contemplation, jeux et sensations nouvelles occupent une place importante dans cette "vie africaine ". La famille sera contrainte de retourner en France après la retraite du médecin. Ainsi, la destination de voyage lui permet de découvrir un nouveau cadre existentiel favorable où il vit les moments les plus intenses de sa vie, loin de la guerre: "C'est ici, dans ce décor que j'ai vécu les moments de ma vie sauvage, libre, presque dangereuse. Une liberté de mouvement, de pensée et d'émotion que je n'ai jamais connue ensuite » (L'A : 24). Autant dire que le voyage pour l'Afrique s'accompagne d'un sentiment de bonheur né du contact sensoriel avec le pays d'accueil. En attestent ces propos du narrateur :

Les jours d'Ogoya étaient devenus mon trésor, le passé lumineux que je ne pouvais pas perdre. Je me souvenais de l'éclat sur la terre rouge, le soleil qui fissurait les routes, la course pieds nus à travers la savane jusqu'aux forteresses des termitières, la montée de l'orage le soir, les nuits bruyantes, criantes, notre chatte qui faisait l'amour avec les tigrillos sur le toit de tôle (L'A : 24-25).

6 Tout comme dans Onistha, les personnages sont contraints à un retour précipité en Europe, ce qui les oblige à délaisser ce nouveau territoire, lieu de leur renaissance.

7 Finalement, les personnages des récits de Le Clézio sont en perpétuel déplacement. Ils vont d'un territoire à un autre : de l'Europe à l'Afrique, puis de l'Afrique pour l'Europe. Se dessine alors un itinéraire spatial du voyage qui se laisse appréhender par la diversité des espaces. Ainsi, lire Onitsha et L'Africain c'est reconstituer au fil des lignes la cartographie des lieux traversés, visités ou habités par les voyageurs. Ayant débarqué au port de Nice le 14 mars 1948, les protagonistes d'Onistha arrivent à destination finale, le 13 avril, non sans avoir fait escale dans la plupart des places portuaires de la côte ouest africaine. Ces arrêts ponctuels permettent la traversée de divers espaces dont rend compte le narrateur en insistant, dans cet extrait, sur la toponymie :

C'était la nuit, on allait vers d'autres ports, Freetown, Monrovia, Takoradi, Cotonou, (...) On allait vers Takoradi, Lomé, Cotonou, on allait vers Conakry, Sherbro, Lavannah, Edima, Manna, Sinou, Accra, Bonny, Calabar (...) On allait vers d'autres ports, d'autres embouchures. Manna, Setta, Krous, Tabu, Sassandra, (...) Volta, Lagos, Victoria, etc. $(0: 40-46)$. (...) Il [Le Surubaya] glissait le long des quais, il allait vers la passe, vers Cape Coast, Accra, Keta, Lomé, Petit Popo, on allait vers l'estuaire $\mathrm{du}$ grand fleuve Volta, vers Cotonou, Lagos, vers l'eau boueuse du fleuve Ogun, vers les bouches qui laissaient couler un océan de boue, à l'estuaire du fleuve Niger $(0$ : 53).

8 Si ces lieux sont traversés par les voyageurs, d'autres sont plutôt habités. Il s'agit notamment de la destination finale Onistha où la famille Allen séjourne durant plusieurs années. Cette même propension à dresser une cartographie des espaces vécus 
est également présente dans L'Africain. Au-delà des lieux évoqués et traversés, le narrateur dresse une carte des quartiers et villes voisines de Banso et d'Ogoja. Cette toponymie excessivement détaillée va jusqu'à trahir la familiarité du narrateur avec ces lieux, ce qu'on note dans cet extrait :

C'est en écrivant que je comprends, maintenant. Cette mémoire n'est pas seulement la mienne. Elle est aussi celle du temps qui a précédé ma naissance, lorsque mon père et ma mère marchaient ensemble sur les routes du haut pays, dans les royaumes de l'ouest du Cameroun (...) Alors ils allaient dans la liberté des chemins, et les noms de lieux sont entrés en moi comme des noms de famille, Bali, Nkom, Bamenda, Banso, Nkongsamba, Revi, Kwaja. Et les noms des pays, Mbembé, Kaka, Nsungli, Bum, Fungom (L'A : 122-123).

Comme on le voit, la géo/graphie, cette écriture de l'espace se mue en une litanie des noms des lieux parcourus par le voyageur. En ce sens, on constate qu'écrire c'est "énumérer des noms. C'est comme un voyage où le fil de la vie collecte les petites perles géographiques» (Andrzej Stasiuk cité par Westphal, $2007: 190)$. Autant dire que «le voyageur ne se cantonne plus dans le seul spectacle sensible du monde. Il rend compte de la qualité abstraite des espaces qu'il parcourt; il instaure une véritable réflexion sur la nature des espaces humains " (Westphal, 2007: 46). Pour parachever cette représentation pittoresque, Le Clézio accompagne les descriptions hyperréalistes et la toponymie avec les noms des différents peuples qui habitent les contrées évoquées: "Les gens d'Akwa [et] les gens d'Adamawa» ( $0: 138)$, «les Yorubas, les Ibos » $(0: 239)$, « les Peuls, des Ouolofs, des Mandingues... » $(0: 42)$, « les Haoussas de Kano » $(0: 113)$, les peuples des grassfields, les Yao, les Banso ( $L ' A: 83)$, entre autres.

Succinctement, les voyageurs de Le Clézio, en quête de survie, sont contraints de quitter les espaces occidentaux dysphoriques pour des milieux africains euphoriques, dans le but de rechercher de nouveaux repères, voire de recommencer une nouvelle vie. Cet établissement reste provisoire puisqu'ils sont obligés de revenir en Europe. Ce retour est difficile car les voyageurs ont du mal à se séparer des espaces où ils ont trouvé du bonheur. Se dessine alors un itinéraire du voyage de l'Europe pour l'Afrique puis de l'Afrique pour l'Europe, itinéraire qui ouvre la voie à de nombreuses découvertes.

\section{Des découvertes et rencontres particulières}

11 Comme nous venons de le démontrer, le voyage suppose un déplacement d'un lieu à un autre. Au cours de celui-ci, les voyageurs rendent compte de leurs émotions à travers les choses vues, ressenties et entendues. Ils s'émerveillent et s'enthousiasment de toutes les expériences vécues. Voyager c'est donc découvrir la diversité et la richesse des peuples rencontrés. Autant dire que «le seul véritable voyage (...), ce ne serait pas d'aller vers de nouveaux paysages, mais d'avoir d'autres yeux, de voir l'univers avec les yeux d'un autre, de cent autres " (Proust, $1988:$ 762). Dans les récits, les voyageurs découvrent, avec passion l'espace, la liberté, les cultures. Les personnages le cléziens sont émerveillés par l'espace africain. En effet, après avoir quitté la France caractérisée par la promiscuité et l'étouffement dus à la guerre, ils arrivent en Afrique où ils trouvent un immense espace. Le climat, le relief, la végétation et les précipitations y sont des attractions majeures. Autant de sensations qui laissent des marques indélébiles et nourrissent leur vie quotidienne à Onitsha et à Banso. Parlant de Fintan, le narrateur d'Onitsha écrit : 
Il n'avait jamais vu tant d'espace. Ibusun, la maison de Geoffroy, était située en dehors de la ville, en amont du fleuve, au-dessus de l'embouchure de la rivière Omerun, là où commençaient les roseaux. De l'autre côté de la butte, vers le soleil levant, il y avait une immense prairie d'herbes jaunes qui s'étendaient à perte de vue $(0: 77)$.

12 C'est à un éloge de la nature pure que s'adonne le voyageur. Celle qui n'a jamais connu les conséquences de l'avancée technologique, encore moins les effets destructeurs des activités humaines.

13 Le corollaire de la sensation que crée l'immensité de l'espace est la licence que s'autorisent les personnages. À l'opposé d'un monde qui les privait de toute liberté, le voyage pour l'Afrique s'apparente à un moment de liberté, voire de libertinage tant les personnages s'adonnent à toutes leurs passions. L'arrivée en Afrique pour le jeune Fintan, tout comme pour le narrateur anonyme, est le symbole de la libération des contraintes vécues à Nice, dans l'appartement de la tante Aurélia. Fintan se lève dès l'aube pour marcher sur des sentiers à peine visibles $(0: 77)$. Chez sa mère Maou, la sensation de liberté est la même. Le narrateur est explicite à ce sujet :

[Maou et Fintan] ont couru jusqu'à la mer, sans même enlever les chaussures, jusqu'à ce que l'eau mousseuse glisse entre les jambes. (...) Ensemble ils tombaient dans la vague nouvelle. La robe blanche de Maou collait sur son corps. Elle tenait à la main le chapeau de paille comme si elle l'avait pêché. Jamais elle n'avait ressenti une telle ivresse, une telle liberté $(0: 50-51)$.

14 L'Afrique est donc le symbole de la liberté de marcher, de courir dans de vastes étendues. L'expression la plus forte de cette sensation est celle du narrateur de L'Africain : «C'est ici, dans ce décor, que j'ai vécu les moments de ma vie sauvage, libre, presque dangereuse. Une liberté de mouvement, de pensée et d'émotion que je n'ai plus jamais connue ensuite » (L'A: 24).

On le voit, la nature est un lieu de recueillement et de régénérescence pour le voyageur. Lorsque celui-ci s'aliène dans l'espace occidental, il trouve un certain réconfort au contact des espaces naturels africains. Se dégage, en filigrane, l'idée d'une certaine affinité entre homme et nature si tant est que, selon les propos de Schoentjes, le «bienêtre particulier éprouvé au contact de la nature est sans doute lié au fait que nous possédons avec le reste du monde vivant une affinité biologique résultant de notre commune évolution» (Schoentjes, 2015: 36). Ainsi par leur retour à la nature, les voyageurs développent une certaine biophilie; les espaces naturels ayant « des effets régulateurs sur [leurs] fonctions vitales" (Ibid.). Autant dire que l'expérience de la nature par le biais du voyage se mue aussi et avant tout en une expérience de soi, dans la mesure où les sujets qui la vivent et y évoluent développent des sentiments puissants qui naissent du caractère idyllique qu'offre le paysage. Éprouvant un sentiment de rejet des espaces européens claustrophobes, marqués par des sévices qu'ils imposent, les voyageurs trouvent, en la nature africaine, un exutoire, un lieu compensateur avec la splendeur de sa faune, de sa flore, de son fleuve et de son relief. Cette écriture écologique ou éco/graphie qui laisse percevoir une opposition entre un milieu occidental dysphorique et un cadre naturel africain euphorique, est la preuve que les voyageurs s'émerveillent à la découverte des lieux qu'ils traversent et qui les traversent.

16 Au-delà de la découverte des espaces naturels africains, le voyage permet également de faire des rencontres extraordinaires avec ce peuple. Il s'agit notamment de son histoire et de ses mentalités. Commençons d'abord par la passion pour l'histoire. Elle est plus 
intense dans Onitsha où Geoffroy Allen est presque obsédé par celle du peuple de Méroë. Les légendes, les mythes et les symboles de ce peuple passionnent le personnage au point qu'il ne veut pas quitter cette ville, même s'il est renvoyé de son service. On dirait que l'histoire du peuple de Méroë lui inspire une poétique de l'existence. Ayant quitté l'Europe pour l'exercice de sa profession, il manifeste son intérêt pour le mythe de Méroë dont le narrateur rend compte :

Partir, pour l'Égypte, pour le Soudan, pour aller jusqu'à Méroë, suivre cette trace. Il ne parlait que de cela, du dernier royaume du Nil, de la reine noire qui avait traversé le désert jusqu'au cœur de l'Afrique. Il parlait de cela comme si rien du monde présent n'avait d'importance, comme si la lumière de la légende brillait plus que le soleil visible (...) Geoffroy Allen était parti tout de suite pour l'Afrique de l'Ouest, sur le fleuve Niger $(0: 84-85)$.

17 En effet, ce peuple avait quitté l'Egypte, chassé par la guerre. Tout au long de son parcours, il a connu des tragédies, a affronté l'hostilité des climats. Mais guidé par une Reine aux pouvoirs surnaturels et de grands prêtres, il a pu atteindre la terre promise et dont Geoffroy entend retrouver les traces. Ses recherches, déclenchées par le guide géographique, le mènent à la découverte des légendes, des mythes, des croyances et de la culture de ce peuple. Il se sert de la carte dressée par Ptolémée pour rechercher le chemin suivi par ce peuple dont il fait une étude anthropologique :

Dans le bureau de Geoffroy, Fintan avait vu un grand dessin épinglé au mur, une carte, qui représentait le Nil et le Niger. En haut de la carte, il y avait écrit PTOLÉMAïs, et partout, des noms étranges, AMMON, Lac de Lyoconède, Garamantiké, Pharax, Mélanogaitouloï, Geïra, Nigeira Metropolis. Entre les fleuves était tracée au crayon rouge la route qu'avait suivie la reine de Meroë, quand elle était partie à la recherche d'un autre monde avec tout son peuple $(0: 119)$.

Le médecin de L'Africain est aussi bien imprégné de l'histoire du Cameroun. Il a passé plus de vingt ans dans la localité d'Ogoja sans aller en Europe. Ainsi peut-on dégager du texte une bonne tranche de l'histoire de ce pays, surtout sa répartition administrative avant les indépendances du Cameroun et du Nigéria. D'ailleurs, il entretient des liens forts avec un personnage historique : il s'agit d'Ahmadou Ahidjo, premier président du Cameroun indépendant qui était son meilleur ami.

Par ailleurs, le séjour des voyageurs en Afrique leur a permis d'entrer en contact avec les mentalités des peuples locaux. Bien qu'ils soient retournés en France, ceux-ci n'oublient pas pour autant les nouvelles rencontres avec l'Altérité. Le voyage a été pour eux une source de connaissance et d'échange avec des peuples à la culture différente et aux mentalités spécifiques. Ces pratiques nouvelles à leur égard, les voyageurs vont les intégrer dans leur socialisation africaine. Ils n'oublient pas pour autant les expériences acquises dans ces espaces, après leur retour à la terre natale. Dans la communauté culturelle découverte par les voyageurs, le peuple a une relation particulière aux choses. De la gastronomie au domaine vestimentaire, en passant par la symbiose avec l'environnement, voilà autant de pratiques qui les fascinent.

Dans la mentalité de ce peuple, les animaux sont sacrés. Les habitants leur vouent un culte parce qu'ils sont des divinités qui guident leurs pas. Ainsi, sont-ils considérés comme des protecteurs de l'homme. Si le dieu se sent menacé, l'individu appartenant à cette communauté se doit d'interagir en sa faveur. Réputé être un bon chasseur, Bony, un autochtone et ami de Fintan, sait qu'il y a des espèces animales interdites, car elles incarnent des dieux. Ainsi, le faucon, tout comme la mante, symbolisent des divinités protectrices de la collectivité dont la filiation se rattache à l'ancêtre fondateur Méroë. 
De ce fait, ils cessent d'être de simples animaux dans l'imaginaire de ce peuple, pour incarner des dieux, puisqu'ils intègrent « le mythe fondateur " (Glissant, 1996:62) de la communauté autochtone qui leur consacre d'ailleurs des rites d'adoration. En ce sens se profile derrière les attitudes du jeune Bony une mentalité fondée sur l'harmonie entre l'homme et la faune dont la maladroite violation par l'étranger Geoffroy constitue un sacrilège. Lorsqu'un phénomène semble hors du commun, il est aussitôt associé à une intervention divine. À son écoute, les autochtones accomplissent alors des actes sacrificiels dont le narrateur d'Onitsha rend compte en ces termes :

La nuit, il se passait des choses bizarres, effrayantes. On ne savait pas quoi, on ne voyait pas, mais ça rodait autour de la maison, ça marchait au dehors, dans les herbes, et plus loin, du côté de la pente, dans les marécages de l'Omerun. Bony disait que c'était Oya, la mère des eaux. Il disait que c'était Asaba, le grand serpent qui vit dans les failles, du côté du soleil levant. Il fallait leur parler, à voix basse, dans la nuit, et ne pas oublier de laisser un cadeau, caché dans l'herbe, sur une feuille de plantain, des fruits, du pain, même de l'argent $(0: 90)$.

La célébration de la fête de l'igname est, pour ce peuple, une obligation rituelle impliquant un sacrifice. Selon la genèse du peuple Ibo, le premier habitant du peuple, Eze Ndri, vivait dans une famine sévère. Las de sa situation, il appela en aide le dieu Chuku, afin que celui-ci lui accordât sa grâce en lui donnant de quoi vivre. Le dieu lui posa la condition de tuer ses deux premiers nés. S'il hésite d'abord, la famine allant croissant, Eze Ndri s'exécute. Il donne la mort à ses deux enfants qu'il enterre selon la volonté du dieu Chuku. Au bout de «trois semaines de quatre jours, (...) les jeunes pousses apparurent sur les tombes. Sur la tombe de son fils aîné, Ndri déterra une igname. Il la fit cuire et la mangea, et c'était excellent » $(0: 102)$. Depuis ce jour, la nourriture est devenue abondante. La fête de l'igname devient alors un rituel annuel au cours duquel la population offre à manger (l'igname) au dieu Eze Enu, en guise de reconnaissance: «On lui donne la première igname, bien blanche, sur le linge blanc étendu sur la terre. Sur le linge on place une plume d'aigle blanche, une plume de pintade blanche, et l'igname, blanche comme l'écume » $(0: 210)$.

Bien plus, le voyage pour l'Afrique aura permis à ces protagonistes de partager la philosophie locale au sujet de la conception. En effet, selon ce peuple, un enfant « appartient à la terre sur laquelle [il] a été conçu, et non pas à celle sur laquelle [il] voit le jour " $(0: 278)$. C'est la raison pour laquelle, Mariama, l'enfant de Maou, même née en France, après sa conception au Nigéria, appartient « au fleuve au bord duquel elle avait été conçue » $(0: 283)$. La même philosophie de l'appartenance est observable dans L'Africain. Le narrateur en rend compte : "C'est à cette époque [pendant la guerre] que ma mère est tombée enceinte deux fois. Les Africains ont coutume de dire que les humains ne naissent pas du jour où ils sortent du ventre de leur mère, mais du lieu et de l'instant où ils sont conçus » (L'A : 91-92). Même s'il reconnaît qu'aujourd'hui il a fondé une famille et se sent enraciné en d'autres lieux (la France notamment), il n'ignore pas ses origines africaines car, "[s'il n'avait] pas eu cette connaissance charnelle de l'Afrique, [s'il n'avait] pas perçu cet héritage de [sa] naissance, que [seraitil] devenu? » (L'A: 122). À sa conception donc, c'est la mère Afrique qui l'a bercé, nourri. Il s'avère alors impossible pour lui d'oublier ses origines africaines.

En résumé, les voyageurs de Le Clézio se montrent attentifs à tout ce qui constitue leur nouvel environnement. Ils sont émerveillés par de nouvelles découvertes et rencontres : les espaces naturels, la liberté qu'offre l'Afrique, la passion pour l'histoire, les mythes et la découverte de nouvelles mentalités. Dans ces conditions, le retour en 
Europe est perçu comme un coup d'arrêt dans cette vie festive. Toutefois, après le retour des protagonistes chez eux, ils ne rompent pas pour autant avec les espaces africains. Par les réminiscences, les souvenirs, ils essaient de retrouver du réconfort. La nostalgie subséquente du paradis perdu, nostalgie causée par le regret obsédant d'un lieu et d'un vécu révolus, influe sur leur identité.

\section{Voyage, nostalgie et (re)configuration identitaire} Jankélévitch, un étranger, un exilé qui est « en même temps ici et là-bas, ni ici ni là-bas, présent et absent » (Ibid.). Il se situe à la croisée des chemins, entre un passé heureux révolu et un présent difficile, un espace euphorique perdu et un cadre dysphorique présent. Il se pose le véritable problème du comment vivre ici après avoir été là-bas. Le voyageur nostalgique est finalement un être décentré, l'exemple atypique de la paratopie dont parle Maingueneau, puisqu'il évolue dans une « localité paradoxale (...) qui n'est pas l'absence de tout lieu, mais une difficile négociation entre le lieu et le nonlieu, une localisation parasitaire qui vit de l'impossibilité même de se stabiliser " (Maingueneau, 2004: 53). Il en découle des mutations sur l'identité du voyageur qui, ayant séjourné dans divers espaces, finit par être attentif aux pratiques, pensées et mentalités nouvelles. Ces altérations sont dues au bonheur et à la fascination qui ont accompagné sa rencontre avec l'Altérité. La nostalgie, liée à la perte des choses extraordinaires rencontrées ailleurs et qui l'habitent sous la forme obsessionnelle des souvenirs, contribue à modifier sa perception du monde. Autant dire qu'il se construit au gré de ses différentes rencontres, des espaces qu'il a traversés et qui l'ont traversé. Ceux-ci lui dictent un ensemble de valeurs et de principes, de règles de conduite, tant son intégration en un autre lieu, suite au voyage, tend à remettre en cause les acquis du lieu de départ. En cela, il devient un apatride, puisqu'il ne peut plus revenir ici en faisant fi de ce qu'il a connu et vécu ailleurs. Il devient alors un être a-centré ou décentré sans repère unique.

L'identité de Fintan est modifiée par son voyage. En effet, ayant débarqué en Afrique à la demande de son géniteur, il s'adapte aussitôt arrivé à Onitsha. Encore en France, il nourrit beaucoup d'affection pour cette localité africaine qu'il ne connaît que grâce aux lettres envoyées par son père. Cet amour n'a pas cessé, puisque dans le bateau, il semble impatient d'atteindre la côte africaine. Le voyage qui dure un mois semble freiner son désir. C'est donc un individu attentif à l'altérité qui débarque à Onitsha, ville à laquelle il va rapidement s'adapter. Il noue aussitôt une amitié avec Bony, un autochtone, qui l'initie à un ensemble d'activités : marche à pieds, marche "pieds nus ", utilisation de la terre pétrie pour fabriquer des statuettes représentant les dieux de la cité, domptage des serpents, chasse et interprétation des symboles et des indices. Au sujet de la marche à pieds, le narrateur écrit :

Au début, Fintan avait mis ses grosses chaussures de laine que portaient les Anglais. (...) Mais un jour qu'il n'avait pas regardé où il mettait les pieds, Fintan avait eu les chaussures pleines de fourmis rouges. (...) À partir de ce jour-là, Fintan n'avait plus voulu porter ni chaussettes ni chaussures. Bony lui avait fait toucher la plante de ses pieds, dure comme une semelle de bois. Fintan avait caché les fameuses chaussettes dans son hamac, il avait mis les grosses chaussures noires dans l'armoire métallique, et il avait marché pieds nus à travers les herbes $(0: 79)$. 
Ce changement d'accoutrement de Fintan est la marque d'une altération identitaire, de son adaptation et de son intégration dans cet univers qui ne lui est plus étranger : "Maintenant, il savait faire cela, marcher pieds nus sans craindre les fourmis ou les épines, et suivre une trace à l'odeur, chasser la nuit. Il devinait la présence des animaux cachés dans les herbes, les pintades blotties contre un arbre, le mouvement rapide des serpents, parfois l'odeur âcre d'un chat sauvage " $(0: 180-181)$. Cette formation du personnage est presque fidèlement reprise dans L'Africain où les petits garçons de l'entourage $\mathrm{du}$ narrateur sont ses initiateurs. Le narrateur nous en donne un éclaircissement : « À l'instigation des enfants du village, la bande un peu hétéroclite (...) comportait des tout-petits tout nus avec un gros ventre et des presque adolescents de douze, treize ans, vêtus comme nous d'un short kaki et d'une chemise, et qui nous avaient appris à ôter chaussures et chaussettes de laine pour courir pieds nus dans les herbes » (L'A : 29).

Le jeune Fintan est aussi influencé par d'autres éléments culturels du territoire habité. Il acquiert rapidement les notions de base de la langue pidgin ( $0: 78)$. Il est un adepte des mets que la grand-mère de Bony concocte. Suite à autant d'expériences qui altèrent considérablement son être, Fintan refuse de retourner en Angleterre, car « quelquefois [il] pensait que c'était vraiment sa famille, que sa peau était devenue comme celle de Bony, noire et lisse » $(0: 208-209)$, « qu'il était né ici, près du fleuve » $(0: 184)$. Si le retour est annoncé comme paisible, retour après lequel il devrait tout oublier de sa vie passée, il importe de noter que ce recommencement potentiel est une chimère, une pure illusion :

On partait. On irait à Londres, ou bien en France, à Nice peut-être, pour être plus près de l'Italie. On aurait une autre vie. Fintan irait à l'école. Il aurait des amis de son âge, il apprendrait à jouer à leurs jeux, à rire avec eux, à se battre comme des enfants, sans toucher au visage. Il irait à vélo, à patins, il mangerait des pommes de terre, du pain blanc, il boirait du lait, du sirop, il mangerait des pommes. Il ne mangerait plus du poisson séché, de piment, de plantain, d'okra. Il oublierait le foufou, l'igname rôtie, la soupe d'arachides. Il apprendrait à marcher avec les chaussures, à traverser les rues entre les autos. Il oublierait le pidgin, il ne dirait plus : «Da buk we yu bin gimmi a om los am.» Il ne dirait plus «Chaka!» à l'ivrogne qui titube sur la route de poussière. Il n'appellerait plus « Nana » la vieille Ugo, la grand-mère de Bony. Elle ne l'appellerait plus par ce petit nom doux qu'il aimait; «Umu.» A Marseille, la grand-mère Aurelia peut lui dire à nouveau «bellino », en l'embrassant bien fort, et l'emmener au cinéma. C'était comme s'il n'était jamais parti $(0: 251-252)$.

Même revenu en Angleterre, il ne rompt pas pour autant avec les territoires conquis. Il emporte avec lui ces nombreuses expériences acquises ailleurs. Sa langue est contaminée par le pidgin. Il est obsédé par les souvenirs. Ceux-ci deviennent presque obsessionnels puisqu'il prend bien le soin d'établir des comparaisons entre les expériences vécues dans d'autres lieux et celles connues en Afrique. Autant dire que son identité se construit sur la diversité des espaces habités ou traversés.

Contrairement à son fils qui s'adapte rapidement à Onitsha, Maou a du mal à se familiariser avec cet univers qu'elle avait trop rêvé. Elle noue tout de même des relations avec Marima, la femme d'Elijah et avec Oya, la reine des eaux. Celles-ci l'initient aux jeux et à la langue du terroir. Avec Mariama, " Maou apprenait des mots dans sa langue. Ulo, la maison. Mmiri, de l'eau. Umu, les enfants. Aja, chien. Odeluede, c'est doux. Je nuo, boire. Ofee, jamais ça; So! Parle! Tekateka, le temps passe... » $(0: 170)$, tandis qu'avec Oya, elle «cherchait à lui parler avec le langage des gestes » $(0: 172)$. 
Elle partage ainsi les mêmes valeurs que ces autochtones qu'elle a rencontrés. Le récit prend des allures didactiques, le lecteur apprend, en même temps que les personnages, les rudiments de vie dans cette nouvelle société. Par la langue comme par de nouvelles habitudes vestimentaires $(0: 99,171)$ ou par la gastronomie $(0: 187,210,243)$ se manifeste l'altération provoquée sur Maou par le voyage. Comme le constate Catherine Kern, «ces scènes d'apprentissage sont avant tout des scènes d'ouverture, partage d'une culture, accueil de l'étranger.» (Kern, 2007) Même si cet apprentissage interculturel est stoppé avec la révocation de Geoffroy Allen, révocation qui marque un retour aux origines, il n'en demeure pas moins que c'est une femme autre qui rentre en Europe. Influencée par son séjour africain, elle « était devenue quelqu'un d'autre. [...] Maintenant, elle appartenait au fleuve, à cette ville. Elle connaissait chaque rue, chaque maison, elle savait reconnaître les arbres et les oiseaux, elle pouvait lire dans le ciel, deviner le vent, entendre chaque détail de la nuit » $(0: 148)$.

Geoffroy Allen, lui aussi, est présenté à son retour comme étant « devenu un autre homme, un étranger» (1991: 147-148). Tout comme lui, le père du narrateur dans L'Africain est un voyageur qui ne cesse de penser au pays d'accueil. Après tout ce temps passé sur les fleuves, il ne peut pas revenir en Europe « où il se [sent] à l'étroit au milieu des gens égoïstes et vaniteux" (L'A: 62). Quand bien même il devra le quitter, il l'emportera comme une marque indélébile :

L'Afrique avait mis en lui une marque qui se confondait avec les traces laissées par

l'éducation spartiate de sa famille à Maurice. L'habit à l'occidental qu'il endossait chaque matin pour aller au marché devait lui peser. Dès qu'il rentrait chez lui, il enfilait une large chemise à la manière des tuniques des Haoussa du Cameroun, qu'il gardait jusqu'à l'heure de se coucher (L'A : 66-67).

31 Revenu en France pour sa retraite, il est « un vieil homme dépaysé, exilé de sa vie et de sa passion » (L'A: 67). Il est un être influencé par certaines manières africaines qui sont devenues sa seconde nature car, "c'était la voix de l'Afrique qui parlait en lui, qui réveillait ses sentiments anciens " (L'A :112-113).

En observant la trajectoire des voyageurs le cléziens, on peut donc dire qu'ils se construisent en fonction des espaces humains et naturels où ils ont séjourné. La rencontre avec l'autre fait d'eux des conciliateurs qui peuvent s'initier aux valeurs de l'Autre pour intégrer la société et renaître. L'originalité de ces personnages réside dans le fait qu'ils semblent oublier leur pays d'origine, tant ils sont fascinés par les pratiques et les manifestations culturelles extraordinaires découvertes ailleurs. Autant dire avec Ngetcham que «le voyage peut alors apparaitre comme le catalyseur par lequel l'individu se (re)construit ou se réalise, parfois en rupture avec ses origines " (Ngetcham, 2013). Cette construction de soi qui se situe entre « la désappartenance et l'appartenance à tous [est la marque d'une] fluidité identitaire dans laquelle les personnages transcendent des existences, des chronotopes de la différence et apparaissent comme des figures de médiations entre les communautés et les cultures " (Mbassi Ateba, $2008: 349$ ). Pour tout dire, le voyage permet une reconfiguration de soi.

\section{Conclusion}

La présente réflexion avait pour but d'analyser les modalités du voyage dans L'Africain et Onitsha de J.M.G Le Clézio. Quittant l'Europe à cause de son inconfort, les voyageurs s'installent en Afrique où ils s'ancrent fermement. Les détails climatiques et 
géographiques, les jeux, l'adaptation aux mets et aux objets culturels, l'intérêt porté à l'histoire du peuple mis en scène, aux mentalités, toutes ces découvertes et expériences façonnent leur identité. Revenus dans leur territoire de départ, ils ne parviennent pas à se séparer de ce passé africain qui les obsède. La nostalgie d'un passé heureux révolu prend alors le pas sur un présent médiocre.

\section{BIBLIOGRAPHIE}

CLÉZIO(Le), Jean-Marie Gustave (1991). Onitsha. Paris : Folio.

CLÉŻo (Le), Jean-Marie Gustave (2004). L’Africain. Paris : Folio.

GLISSANT, Édouard (1996). Introduction à une poétique du divers. Paris : Gallimard.

JANKELEVITCH, Vladimir (1974). L'Irréversible et la nostalgie. Paris : Flammarion.

KERN, Catherine (2015). « J.M.G. Le Clézio, écrivain de l'Afrique », Semen [En ligne] URL : http://

semen.revues.org/2250, page consultée le 24 mars 2015.

MAINGUENEAU, Dominique (2004). Discours littéraire : paratopie et scènes d'énonciation. Paris : Armand Colin.

MBASSI ATEBA, Raymond, (2008). Identité et fluidité dans l'œuvre de Jean-Marie Gustave Le Clézio. Une poétique de la mondialité. Paris : L'Harmattan.

NGETCHAM (2013). «Quand le voyage(ur) s'écrit dans Le Rat d'Amérique de Jacques Lanzmann, L'Africain et Onitsha de Jean-Marie Gustave Le Clézio ", communication au colloque « Voyages ", $\mathrm{X}^{\mathrm{e}}$ colloque international d'Études francophones, CIEFT à Timisoara (Roumanie), les 15 et 16 mars 2013.

PROUST, Marcel (1988). La Prisonnière, dans À La Recherche du Temps Perdu. Paris : Ed. Gallimard, Bibliothèque de la Pléiade.

SCHOENTJES, Pierre (2015). Ce qui a lieu. Essai d'écopoétique. Paris : Éditions Wildproject.

WESTPHAL, Bertrand (2000). La Géocritique mode d'emploi. Paris : Presses universitaires de Limoges,

«Espaces humains ».

WESTPHAL, Bertrand (2007). La Géocritique : réel, fiction, espace. Paris : Minuit.

\section{NOTES}

1. Dans la suite de notre réflexion, nous les abrégerons respectivement $L^{\prime} A$ et $O$ suivis du numéro de la page citée. 


\section{RÉSUMÉS}

À l'aune de la géocritique, la présente étude analyse la trajectoire des personnages nostalgiques dans Onitsha et L'Africain de J.M.G Le Clézio. Fuyant les malaises existentiels dans leur lieu d'origine, les personnages partent pour un ailleurs (l'Afrique). Conçu au départ comme un déplacement avec idée de retour, le voyage se mue en quête de soi. Influencés par les rencontres avec l'altérité africaine qui a fasciné et façonne leur identité, les voyageurs ont du mal à se départir de cet espace. Se dessine alors un itinéraire du voyage par lequel les personnages manifestent leur nostalgie du paradis perdu.

In the lights of geocriticism, this study analyzes the trajectory of nostalgic characters in Onitsha and L'Africain by J.M.G Le Clézio. Fleeing existential discomfort in their place of origin, the characters leave for somewhere else (Africa). Originally designed as a travel with the idea of returning, the journey turns into a quest for oneself. Influenced by encounters with African otherness that have fascinated and shaped their identity, travelers find it difficult to divest themselves of this space. An itinerary of the journey is then drawn by which the characters express their nostalgia for the lost paradise.

\section{INDEX}

Mots-clés : voyage, espace, découverte, nostalgie, Le Clézio (Jean-Marie Gustave)

Keywords : journey, spaces, discoveries, nostalgia, Le Clézio (Jean-Marie Gustave)

\section{AUTEUR}

\section{MOUHAMADOU NGAPOUT KPOUMIÉ}

Université de Dschang (Cameroun)

ngapout.mouhamadou[at]yahoo.fr 